\title{
ChemComm
}

\section{Rational design and diversity-oriented synthesis of peptoid-based selective HDAC6 inhibitors $\uparrow$}

Cite this: Chem. Commun., 2016, 52, 3219

Received 15th December 2015 Accepted 14th January 2016

DOI: $10.1039 / \mathrm{c} 5 \mathrm{cc} 10301 \mathrm{k}$

www.rsc.org/chemcomm

A mini library of HDAC inhibitors with peptoid-based cap groups was synthesized using an efficient multicomponent approach. Four compounds were identified as potent HDAC6 inhibitors with a selectivity over other HDAC isoforms. The most potent HDAC6 inhibitor revealed remarkable chemosensitizing properties and completely reverted the cisplatin resistance in Cal27 CisR cells.

Histone deacetylases (HDACs) catalyze the cleavage of acetyl groups from $\mathrm{N}$-acetyl-lysine residues of histones and non-histone proteins. These posttranslational modifications (PTMs) are important for the regulation of gene transcription and protein function. ${ }^{1}$ HDAC classes I (HDACs 1-3,8), IIa (HDACs 4,5,7,9), IIb (HDACs 6,10), and IV (HDAC 11) contain zinc-dependent deacetylase domains and are considered "classical" HDACs. ${ }^{2,3}$ The class III HDACs (sirtuins) are structurally different and $\mathrm{NAD}^{+}$-dependent. ${ }^{3}$ Most inhibitors of "classical" HDACs are characterized by a widely accepted pharmacophore model comprising a zinc binding group (ZBG) chelating the zinc atom in the active site, a linker accommodating the tubular access of the active site, and a cap group interacting with the external surface (Fig. 1A). ${ }^{4}$ Currently, four HDAC inhibitors (HDACi) have been approved by the FDA for the treatment of cancer (vorinostat, romidepsin, belinostat and panobinostat). Furthermore, HDACi have attracted attention as potential therapeutic drugs for treating a variety of diseases beyond cancer including inflammation, diabetes, HIV, neurodegenerative diseases (e.g. Alzheimer, Huntington), and parasitic diseases (e.g. malaria). ${ }^{5}$

All approved HDACi target multiple HDAC isoforms. Their clinical use may therefore cause serious unwanted side effects. ${ }^{3}$

\footnotetext{
${ }^{a}$ Institut für Pharmazeutische und Medizinische Chemie, Heinrich-Heine-Universität Düsseldorf, Universitätsstr. 1, 40225 Düsseldorf, Germany. E-mail: finn.hansen@hhu.de

${ }^{b}$ Institut für Anorganische Chemie und Strukturchemie, Heinrich-Heine-Universität Düsseldorf, Universitätsstr. 1, 40225 Düsseldorf, Germany

$\dagger$ Electronic supplementary information (ESI) available: Experimental details (synthetic protocols, bioassays, X-ray crystallography, computational methods), characterization data of new compounds, and copies of NMR spectra. CCDC 1434499. For ESI and crystallographic data in CIF or other electronic format see DOI: $10.1039 / \mathrm{c} 5 \mathrm{cc} 10301 \mathrm{k}$
}

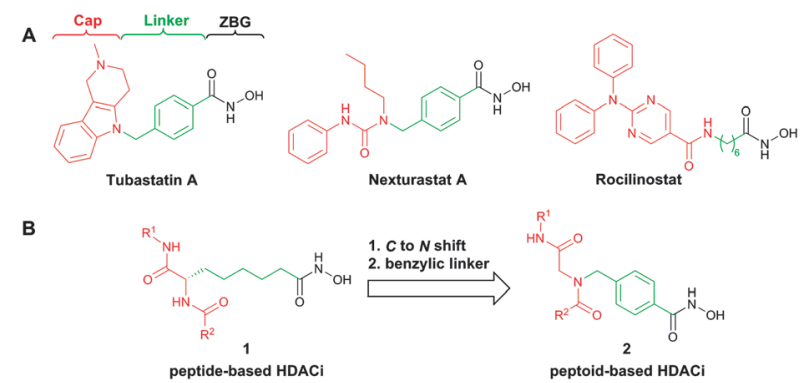

Fig. 1 (A) Selected HDAC6-selective HDACi; (B) design of peptoid-based HDACi.

Thus, the development of isoform-selective HDACi has been hypothesized to afford inhibitors with an improved safety profile., HDAC6, a class IIb enzyme, is structurally and functionally unique among the eleven human zinc-dependent HDACs. ${ }^{6}$ It is the only HDAC with two independent functional catalytic domains and a C-terminal zinc finger motif responsible for binding ubiquitinated proteins. ${ }^{5 b}$ Interestingly, it has been demonstrated that the second catalytic site is the major functional domain of HDAC6. ${ }^{7}$ The enzyme was initially described as tubulin deacetylase; however, it also modulates the function of other non-histone proteins implicated in regulatory processes, including cortactin, peroxiredoxins, and Hsp90. ${ }^{5 b}$ Due to the large number of substrates, HDAC6 is involved in numerous diseases such as autoimmune disorders, inflammation, neurodegenerative diseases, and cancer. ${ }^{5 b}$ Notably, the first selective HDAC6 inhibitor, rocilinostat (ACY-1215, Fig. 1A), has recently entered phase II clinical trials for the treatment of multiple myeloma and lymphoid malignancies. ${ }^{5 a}$ Consequently, HDAC6 has emerged as an attractive therapeutic target, and the search for novel potent and selective HDAC6 inhibitors is of high importance.

We report here on the rational design and diversity-oriented synthesis of a series of selective HDAC6 inhibitors utilizing peptoidbased cap groups. The biological evaluation of the target compounds includes whole cell HDAC and MTT assays on sensitive and chemoresistant cancer cell lines. The most active peptoid-based 
HDACi were investigated for their activity against selected HDAC isoforms and their enhancement of cisplatin-induced cytotoxicity. NMR spectroscopy and X-ray crystallography were employed to study the peptoid amide bond geometry. Molecular modelling, MD simulations, and docking studies allowed rationalization of the observed selectivity profile.

Recently, it has been identified via homology modelling that the catalytic domain II (CDII) of HDAC6 possesses a significantly wider channel rim in comparison with HDAC1. ${ }^{8 a}$ This feature has been used to develop selective HDAC6 inhibitors utilizing bulky or branched cap groups. ${ }^{8}$ Tubastatin A and nexturastat A (Fig. 1A) are important examples that occupy this unique rim region of HDAC6. ${ }^{8}$ Fairlie and co-workers previously described peptide-based HDACi utilizing 2-aminosuberic acid as key building block. ${ }^{9}$ These HDACi of type 1 (Fig. 1B) revealed remarkable anticancer activity. Notably, some compounds of this type showed a preference for HDAC6. ${ }^{10}$ However, peptides are intrinsically labile to enzymatic degradation, and the large scale synthesis of enantiomerically pure unnatural amino acids is often a challenging task. Peptoids, $N$-alkyl glycine derivatives, feature several advantages over peptides including proteolytic stability and increased cell permeability. ${ }^{11}$ We hypothesized that peptoid analogues 2 of the $\alpha$-amino suberic acid-containing HDACi (1) having the linker attached to the nitrogen rather than to the $\alpha$-carbon are promising scaffolds for the development of selective HDAC6 inhibitors. The peptoid-based cap group is intended to accommodate the significantly wider catalytic channel rim identified in human HDAC6. Furthermore, we decided to incorporate a benzyl linker, which is known to increase the selectivity for HDAC6 (Fig. 1B). ${ }^{8}$

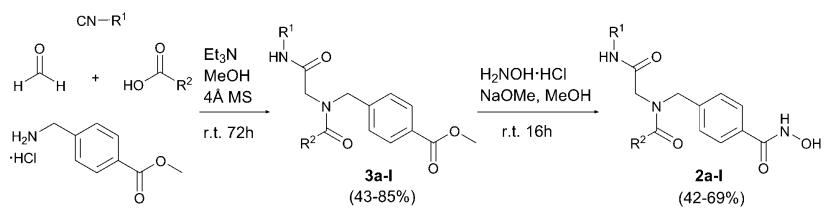

Scheme 1 Synthesis of peptoid-based HDACi $2 a-1$.
We aimed at the development of a multicomponent approach in order to allow a rapid and diversity-oriented synthesis of the target compounds. Our retrosynthetic analysis led to an Ugi fourcomponent reaction (U-4CR) as the key step followed by hydroxylaminolysis as a suitable synthetic pathway. Although the U-4CR has been used previously for the preparation of HDACi, ${ }^{12}$ no synthesis of peptoid-based HDACi via the U-4CR has been reported so far. Preparative conditions for the U-4CR were first optimized using intermediate $3 \mathrm{a}\left(\mathrm{R}^{1}: t\right.$-Bu; $\left.\mathrm{R}^{2}: 3,4-\mathrm{Me}-\mathrm{Ph}\right)$ as model compound (Table S1, ESI $\dagger$ ). Different solvents, stoichiometries, concentrations, and reaction times were studied. Best results were achieved at room temperature using dry methanol as solvent in the presence of $4 \AA$ molecular sieves. This optimized protocol was then used for the synthesis of the intermediates $\mathbf{3 a} \mathbf{a}-\mathbf{l}$. In all cases, the crude products were purified by flash column chromatography and isolated in $43-85 \%$ yield. The subsequent hydroxylaminolysis afforded the desired HDACi 2a-1 in 42-69\% yield (Scheme 1).

All synthesized peptoid-based HDACi $2 \mathbf{a}-\mathbf{l}$ were first assessed in a whole cell HDAC inhibition assay and a MTT assay for cytotoxicity using the human ovarian cancer cell line A2780 and its cisplatin resistant subclone A2780 CisR. The results of both assays are summarized in Table 1 . The whole cell HDAC assay provided valuable structure-activity relationships. Notably, all HDACi with a 4-dimethylamino-substituted cap group (2e, i, l) displayed remarkable activity in the whole cell HDAC assay and showed similar or better potency than the FDA-approved reference compound vorinostat. The most active compound from this series, $2 \mathbf{i}$, showed $\mathrm{IC}_{50}$ values of $0.90 \mu \mathrm{M}$ (A2780) and $0.79 \mu \mathrm{M}$ (A2780 CisR). Interestingly, the nature of the isocyanide component $\left(\mathrm{R}^{1}\right)$ used in U-4CR is important for the activity. The potency of the compounds decreased according to their $\mathrm{R}^{1}$ substituent in the order cyclohexyl $>4$-tolyl $>$ tert-butyl. All compounds (2a-1) showed cytotoxic activities against the ovarian cancer cell lines A2780/A2780 CisR. In good agreement with the data from the whole cell HDAC assay, 2e, i, $\mathbf{l}$ exhibited remarkable anticancer activity and displayed higher cytotoxicity than the reference compound cisplatin. Notably, $2 \mathbf{i}$ was the most potent

Table 1 HDAC inhibition and cytotoxic activity (MTT) of $\mathbf{2 a - l}$ against the human ovarian cancer cell lines A2780 and the human tongue squamous cell carcinoma cell line Cal27 and their cisplatin resistant sublines A2780 CisR and Cal27 CisR

\begin{tabular}{|c|c|c|c|c|c|c|c|c|c|c|}
\hline \multirow[b]{2}{*}{ Entry } & \multirow[b]{2}{*}{$\mathrm{R}^{1}$} & \multirow[b]{2}{*}{$\mathrm{R}^{2}$} & \multicolumn{2}{|c|}{$\mathrm{HDAC}^{\mathrm{IC}_{50}[\mu \mathrm{M}]}$} & \multicolumn{2}{|c|}{ MTT IC $_{50}[\mu \mathrm{M}]$} & \multicolumn{2}{|c|}{$\underline{\text { HDAC } \mathrm{IC}_{50}[\mu \mathrm{M}]}$} & \multicolumn{2}{|c|}{ MTT IC $_{50}[\mu \mathrm{M}]$} \\
\hline & & & A2780 & A2780 CisR & $\mathrm{A} 2780$ & A2780 CisR & Cal27 & Cal27 CisR & Cal27 & Cal27 CisR \\
\hline $2 \mathbf{a}$ & $t-\mathrm{Bu}$ & 3,4-Me-Ph & 5.14 & 4.97 & 3.59 & 9.39 & 2.05 & 3.22 & 14.7 & 10.7 \\
\hline $2 b$ & $t-\mathrm{Bu}$ & 3,5-Me-Ph & 5.99 & 6.16 & 4.86 & 10.7 & 2.89 & 3.83 & 25.3 & 11.7 \\
\hline $2 \mathrm{c}$ & $t$-Bu & $\mathrm{Ph}$ & 7.82 & 4.82 & 32.1 & 53.6 & 7.83 & 10.6 & 37.1 & 36.7 \\
\hline $2 d$ & $t-\mathrm{Bu}$ & 1-Naphthyl & 15.0 & 15.5 & 8.33 & 31.0 & 13.9 & 18.1 & 27.7 & 34.7 \\
\hline $2 e$ & $t-\mathrm{Bu}$ & $4-\mathrm{Me}_{2} \mathrm{~N}-\mathrm{Ph}$ & 1.23 & 1.03 & 0.94 & 1.62 & 1.08 & 0.76 & 2.92 & 3.83 \\
\hline $2 f$ & $c$-Hex & 3,5-Me-Ph & 2.72 & 2.00 & 6.05 & 19.3 & 3.31 & 2.50 & 7.50 & 12.8 \\
\hline $2 \mathrm{~g}$ & $c$-Hex & $\mathrm{Ph}$ & 3.88 & 3.56 & 20.0 & 32.8 & 5.45 & 3.18 & 16.9 & 14.2 \\
\hline $2 \mathrm{~h}$ & $c$-Hex & 1-Naphthyl & 6.03 & 4.47 & 31.2 & 36.5 & 5.23 & 7.51 & 34.1 & 18.0 \\
\hline $2 \mathrm{i}$ & $c$-Hex & $4-\mathrm{Me}_{2} \mathrm{~N}-\mathrm{Ph}$ & 0.90 & 0.79 & 0.34 & 1.45 & 0.62 & 0.48 & 2.04 & 2.70 \\
\hline $2 \mathrm{j}$ & 4-Tolyl & $\mathrm{Ph}$ & 5.51 & 5.03 & 5.87 & 24.3 & 5.00 & 2.99 & 15.8 & 11.8 \\
\hline $2 \mathbf{k}$ & 4-Tolyl & 1-Naphthyl & 8.51 & 8.94 & 6.31 & 25.4 & 3.93 & 3.76 & 19.8 & 12.3 \\
\hline 21 & 4-Tolyl & $4-\mathrm{Me}_{2} \mathrm{~N}-\mathrm{Ph}$ & 0.90 & 1.05 & 0.65 & 3.88 & 0.77 & 0.57 & 2.77 & 3.44 \\
\hline Vorinostat & & & 0.96 & 0.97 & $2.42^{a}$ & $3.12^{a}$ & 0.86 & 0.73 & $2.64^{a}$ & $2.08^{a}$ \\
\hline Cisplatin & & & - & - & 2.25 & 17.2 & - & - & 2.50 & 16.1 \\
\hline
\end{tabular}

${ }^{a}$ Data from ref. $5 d$. 
compound with $\mathrm{IC}_{50}$ values of $0.34 \mu \mathrm{M}(\mathrm{A} 2780)$ and $1.45 \mu \mathrm{M}$ (A2780 CisR).

To extend the investigation of this novel class of HDACi to another pair of solid cancer cell lines, compounds $\mathbf{2 a}-\mathbf{I}$ were tested for HDAC inhibitory and cytotoxic activity in the human tongue squamous cell carcinoma cell line Cal27 and its cisplatin resistant subline Cal27 CisR. We observed similar structure-activity relationships as seen against A2780/A2780 CisR. Again, 2i was the most potent HDACi (Table 1). Interestingly, the compounds showed almost equipotent cellular HDAC inhibition in the respective cancer cell pairs. However, cytotoxic activity (MTT assay) was higher in A2780 than in A2780 CisR whereas the compounds displayed about similar cytotoxicity in Cal27 and Cal27 CisR cells. Thus, resistance mechanisms induced by cisplatin may (A2780 CisR) or may not (Cal27 CisR) modulate cytotoxic effects of these novel HDACi.

Based on their noteworthy activity in the whole cell HDAC assay, compounds $\mathbf{2 e}, \mathbf{f}, \mathbf{i}, \mathbf{l}$ were selected for a detailed biological evaluation. First, they were assessed for their inhibitory activity against recombinant HDAC6. Strikingly, all four compounds were found to be potent HDAC6 inhibitors with $\mathrm{IC}_{50}$ values ranging from $1.59 \mathrm{nM}$ to $11.2 \mathrm{nM}$ (Table 2). In order to investigate the selectivity against other HDAC isozymes, we screened compounds $\mathbf{2 e}, \mathbf{f}, \mathbf{i}, \mathbf{l}$ against representative examples of all other zinc dependent HDAC classes (class I: HDAC2; class IIa: HDAC4; and class IV: HDAC11). The results are presented in Table 2. Compound $2 \mathbf{f}$ and $2 \mathbf{i}$ demonstrated the highest overall selectivity for HDAC6. 2f revealed selectivity indices of 390 against HDAC2, > 893 against HDAC4 and 38 against HDAC11. The most potent HDAC6 inhibitor 2i (HDAC6 IC $_{50}$ : $1.59 \mathrm{nM}$ ) exhibited selectivity indices of 126 against HDAC2, $>6289$ against HDAC4, and 40 against HDAC11. The HDAC6 inhibitory activity of these compounds was further validated by investigation of the acetylation status of $\alpha$-tubulin in Cal27 and Cal27 CisR cells. As expected, the peptoid-based HDACi $2 \mathbf{e}, \mathbf{f}, \mathbf{i}, \mathbf{l}$ and HDAC6-selective tubastatin A are causing $\alpha$-tubulin hyperacetylation whereas class I HDAC-selective entinostat had no effect. These data confirm the inhibition of HDAC6 in a more complex cellular environment (Fig. S1, ESI†).

The 1D NMR spectra of compounds $\mathbf{2 a}-\mathbf{d}, \mathbf{f}-\mathbf{h}, \mathbf{j}-\mathbf{k}$ at room temperature and of $\mathbf{2 e}, \mathbf{i}, \mathbf{l}$ at $-30{ }^{\circ} \mathrm{C}$ (Fig. S2-S4, ESI $\dagger$ ) revealed the presence of two sets of NMR signals. The occurrence of cis/trans amide bond rotamers in peptoids is a well-known phenomenon. ${ }^{11}$ Accordingly, we assumed that the two sets of NMR signals arise from the restricted rotation around the tertiary amide bond.

Table 2 Inhibitory activities of compounds $\mathbf{2 e}, \mathbf{f}, \mathbf{i}, \mathbf{l}$ against HDAC isoforms 2, 4, 6 and $11^{a}$

\begin{tabular}{lllll}
\hline Compound & $\begin{array}{l}\mathrm{HDAC2} \mathrm{IC}_{50} \\
{[\mathrm{nM}]}\end{array}$ & $\begin{array}{l}\text { HDAC4 } \mathrm{IC}_{50} \\
{[\mathrm{nM}]}\end{array}$ & $\begin{array}{l}\mathrm{HDAC6} \mathrm{IC}_{50} \\
{[\mathrm{nM}]}\end{array}$ & $\begin{array}{l}\text { HDAC11 } \mathrm{IC}_{50} \\
{[\mathrm{nM}]}\end{array}$ \\
\hline $\mathbf{2 e}$ & 799 & $>10000$ & 6.52 & 227 \\
$\mathbf{2 f}$ & 4366 & $>10000$ & 11.2 & 421 \\
$\mathbf{2 i}$ & 200 & $>10000$ & 1.59 & 63.2 \\
$\mathbf{2 l}$ & 637 & 4377 & 2.84 & 25.0 \\
& & & & \\
TSA & 18.9 & nd & 3.18 & 17.8 \\
TMP269 & nd & 169 & nd & nd
\end{tabular}

${ }^{a}$ The inhibition of HDAC isoforms was determined by Reaction Biology Corporation (Malvern, PA, USA).
Indeed, variable temperature NMR studies (VT-NMR, Fig. S5 and S6, ESI $\dagger$ ) using $2 \mathbf{f}$ and $2 \mathbf{i}$ as representative examples confirmed the presence of two rotamers. A careful inspection of the ${ }^{1} \mathrm{H}$ NMR spectra revealed a strongly upfield-shifted position of the glycine methylene resonance signals in the case of the major conformers. This upfield shift of the major species might be attributed to shielding by the aryl ring $\left(\mathrm{R}^{2}\right)$, which is only possible for the cis rotamer indicating a cis amide bond preference. ${ }^{13}$ This was further supported by single crystal X-ray crystallography. Diffraction quality crystals of $2 \mathbf{f}$ were obtained by vapor diffusion (see ESI†). The X-ray crystal structure of $2 \mathbf{f}$ confirmed the cis amide bond geometry in the solid state (Fig. S7 and S8, ESI $\dagger$ ).

A docking study with HDAC2, HDAC4, and HDAC6 was performed to rationalize the selectivity profiles of $2 \mathbf{f}$ and $2 \mathbf{i}$. $2 \mathbf{f}$ and $2 \mathbf{i}$ show the highest selectivity for binding to HDAC6 over HDAC2 and HDAC4 (Table 2) but are less selective with respect to HDAC11. Hence, docking to HDAC11 was omitted as the differences to HDAC6 are expected to be less pronounced. Due to the presence of cis/trans rotamers, it is important to consider both amide bond geometries when investigating the binding modes of $2 \mathbf{f}$ and $2 \mathbf{i}$. Thus, both the cis- and trans-rotamers were docked into crystal structures of HDAC2 and HDAC4 and, due to the lack of a crystal structure, homology models of HDAC6 CDII using AutoDock3 in combination with DrugScore ${ }^{14}$ as successfully applied previously. ${ }^{5 d}$ Class IIa HDACs possess a highly conserved histidine residue in the catalytic domain that is rotated away from the binding site. ${ }^{15}$ In class I isoforms, this histidine is replaced by a tyrosine accommodating an "inward" orientation. Since the conformation of the corresponding tyrosine in HDAC6 is unknown, we generated homology models of HDAC6 CDII with Y301 (numbering based on the homology model) flipped-in and -out and subjected them to all-atom molecular dynamics (MD) simulations of $1 \mu$ s length (see ESI $\dagger$ ). After clustering the generated protein conformations, the cluster-representative of the largest cluster was chosen for molecular docking. Comparing the docking results between the respective HDAC structures, a clear trend is apparent. Regardless of which compound and rotamer was docked, the docking energy of the ligands in HDAC6, considering the Y301 flipped-in structure, was on average $1.67 \mathrm{kcal} \mathrm{mol}^{-1}$ more favorable than in other HDAC isoforms (Table S2, ESI $\dagger$ ), in qualitative agreement with the exhibited isoform selectivity (Table 2). As a reason, in the wider channel rim of HDAC6 lined with hydrophobic amino acids, the hydrophobic $\mathrm{R}^{1}$ groups of $2 \mathbf{f}$ and $2 \mathbf{i}$ become more buried than in the other isoforms (see Fig. 2B-D versus Fig. 2A). In the Y301 flipped-in structure of HDAC6, hydrogen bonding between Y301 and the hydroxamic acid moiety of 2 f and $2 \mathbf{i}$ occurs (Fig. 2B-D), leading to more favorable docking energies than in the flipped-out structure (Fig. S9 and Table S2, ESI $\dagger$ ). 2f and $2 \mathbf{i}$ are not able to bind to the HDAC4 isoform, which is also reflected in the docking where only $<5 \%$ of the docking poses chelated the zinc ion irrespective of the rotamer. Thus, no converged docking solution could be identified in HDAC4. Comparing the two HDACi docked to HDAC6 with Y301 flipped-in, 2i shows on average a by $1 \mathrm{kcal} \mathrm{mol}^{-1}$ more favorable docking energy than $\mathbf{2 f}$, in semi-quantitative agreement with observed inhibition activities (Table S2, ESI $\dagger$ ). First, the channel rim can better accommodate the two methyl groups when attached to the aniline nitrogen of $2 \mathbf{i}$ (Fig. $2 \mathrm{~B}$ ) than when attached in meta positions to the phenyl ring of $2 \mathbf{f}$ (Fig. 2D). Second, as a result, the 
A

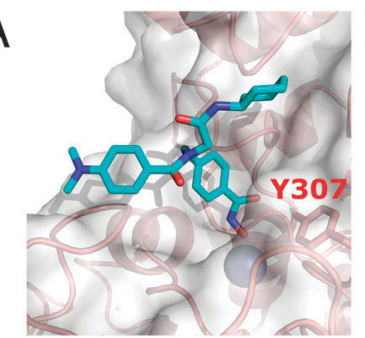

B

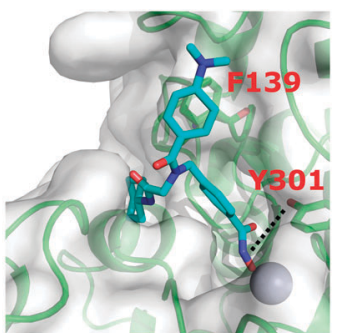

C
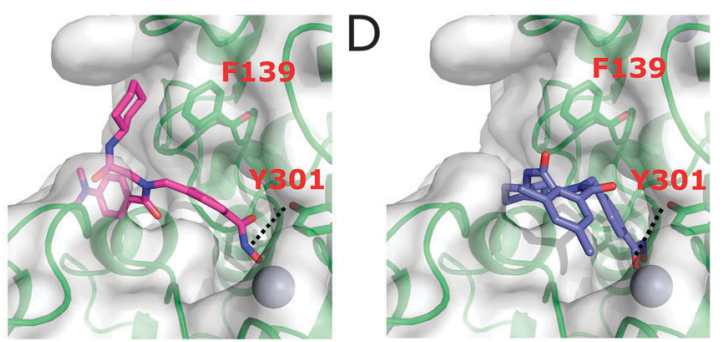

Fig. 2 Docking poses of $\mathbf{2} \mathbf{i}$ as a trans-rotamer (A and B) and cis-rotamer (C), and $\mathbf{2 f}(\mathrm{D})$, to HDAC2 (A) and HDAC6 with Y301 flipped-in (B-D). Zinc is shown as a sphere.

hydrophobic groups of $\mathbf{2 f}$ are less buried than those of $2 \mathbf{i}$, resulting in a lower energetic contribution. The trans-rotamer of $2 \mathbf{i}$ (Fig. $2 \mathrm{~A}$ and B) has on average $1 \mathrm{kcal} \mathrm{mol}^{-1}$ more favorable docking energies than its cis-rotamer (Fig. 2C), as it can form $\pi$-stacking interactions with F139 (Fig. 2B). 2f however does not show an energy difference regarding its two rotamers, in agreement with a less buried binding pose. In summary, the predicted binding modes are in line with and provide structural explanations for the observed selectivity profiles and inhibition activities.

To further study their anticancer properties, compounds $2 \mathbf{e}, \mathbf{f}, \mathbf{i}, \mathbf{1}$ were selected for subsequent drug combination experiments. HDACi might find broad utility as epigenetic primers resensitizing tumours to a primary agent after the tumour has developed resistance. ${ }^{16}$ Thus, we tested compounds $2 \mathbf{2 e}, \mathbf{f}, \mathbf{i}, \mathbf{l}$ in combination with cisplatin using the cell lines Cal27 and the platinum-resistant subline Cal27 CisR. The cells were pretreated with the respective HDACi at a fixed concentration of $1 \mu \mathrm{M}$ for $48 \mathrm{~h}$ followed by a treatment with cisplatin for $72 \mathrm{~h}$. All four compounds markedly enhanced the cisplatin-induced cytotoxicity towards Cal27 and Cal27 CisR (Table 3). The strongest chemosensitizing effect was observed for $2 \mathbf{i}$ showing better shift factors compared with the reference compound tubastatin A. Strikingly, the combination of $2 \mathbf{i}$ and cisplatin led to a complete resensitization of the platinumresistant Cal27 CisR cell line towards cisplatin (shift factor (SF): 5.57) with an $\mathrm{IC}_{50}$ of cisplatin similar to the $\mathrm{IC}_{50}$ in the native cell line Cal27. Thus, $2 \mathbf{i}$ possesses remarkable chemosensitizing properties leading to full reversal of cisplatin resistance in Cal27 CisR.

In conclusion, we have developed a highly efficient multicomponent approach for the diversity-oriented synthesis of peptoid-based HDACi. Our data demonstrate that the peptoidbased HDACi of type 2 are a new class of potent and selective HDAC6 inhibitors with remarkable activity against a panel of cancer cells of different chemosensitivity and tissue origin. Most notably, compound $2 \mathbf{i}$ enhanced cisplatin-induced cytotoxicity and completely resensitized the platinum-resistant Cal27 CisR cell
Table $3 \quad \mathrm{IC}_{50}$ values $(\mu \mathrm{M})$ after treatment of Cal27 and Cal27 CisR with cisplatin alone or in combination with $1 \mu \mathbf{M} \mathbf{2} \mathbf{e}, \mathbf{2} \mathbf{f}, \mathbf{2} \mathbf{i}$, and $\mathbf{2} \mathbf{l}$, respectively. The HDAC6 selective inhibitor tubastatin A (tub A) was included as control. The shift factor (SF) was calculated by dividing the $I_{50}$ of cisplatin alone and the $\mathrm{IC}_{50}$ of the corresponding drug combinations ${ }^{a}$

\begin{tabular}{|c|c|c|c|c|}
\hline \multirow[b]{3}{*}{ Compound } & \multicolumn{4}{|c|}{ Cell line } \\
\hline & \multicolumn{2}{|c|}{ Cal27 } & \multicolumn{2}{|c|}{ Cal27 CisR } \\
\hline & $\mathrm{IC}_{50}$ & $\mathrm{SF}$ & $\mathrm{IC}_{50}$ & $\mathrm{SF}$ \\
\hline Cisplatin & 3.69 & - & 15.7 & - \\
\hline Cisplatin $+2 \mathbf{e}$ & 1.28 & 2.88 & 4.09 & 3.84 \\
\hline Cisplatin $+\mathbf{2 f}$ & 2.17 & 1.70 & 7.72 & 2.03 \\
\hline Cisplatin $+2 \mathbf{i}$ & 0.80 & 4.61 & 2.82 & 5.57 \\
\hline Cisplatin + 21 & 1.15 & 3.21 & 4.40 & 3.57 \\
\hline Cisplatin + tub A & 2.96 & 1.24 & 6.16 & 2.54 \\
\hline
\end{tabular}

${ }^{a}$ Values are the mean of three experiments each performed in triplicate. The standard deviations are $<10 \%$ of the mean.

line towards cisplatin. Taken together, compound 2i represents a valuable lead structure for future efforts towards developing novel HDACi with optimized anticancer properties.

The Deutsche Forschungsgemeinschaft (DFG) is acknowledged for funds used to purchase the UHR-TOF maXis 4G, Bruker Daltonics, Bremen HRMS instrument used in this research. FKH acknowledges financial support from the Fonds der Chemischen Industrie (FCI).

\section{Notes and references}

1 M. Biel, V. Wascholowski and A. Giannis, Angew. Chem., Int. Ed., 2005, 44, 3186.

2 J. E. Bradner, N. West, M. L. Grachan, E. F. Greenberg, S. J. Haggarty, T. Warnow and R. Mazitschek, Nat. Chem. Biol., 2010, 6, 238.

3 O. Witt, H. E. Deubzer, T. Milde and I. Oehme, Cancer Lett., 2009, 277, 8. 4 P. Bertrand, Eur. J. Med. Chem., 2010, 45, 2095.

5 (a) K. Falkenberg and R. Johnstone, Nat. Rev. Drug Discovery, 2014, 13, 673; (b) J. H. Kalin and J. A. Bergman, J. Med. Chem., 2013, 56, 6297; (c) F. K. Hansen, T. S. Skinner-Adams, S. Duffy, L. Marek, S. D. M. Sumanadasa, K. Kuna, J. Held, V. M. Avery, K. T. Andrews and T. Kurz, ChemMedChem, 2014, 9, 665; (d) L. Marek, A. Hamacher, F. K. Hansen, K. Kuna, H. Gohlke, M. U. Kassack and T. Kurz, J. Med. Chem., 2013, 56, 427; (e) R. De Vreese, T. Verhaeghe, T. Desmet and M. D'hooghe, Chem. Commun., 2013, 49, 3775.

6 J.-H. Lee, A. Mahendran, Y. Yao, L. Ngo, G. Vente-Perez, M. L. Choy, N. Kim, W.-S. Ham, R. Breslow and P. A. Marks, Proc. Natl. Acad. Sci. U. S. A., 2013, 110, 15704.

7 H. Zou, Y. Wu, M. Navre and B. C. Sang, Biochem. Biophys. Res. Commun., 2006, 341, 45.

8 (a) K. V. Butler, J. Kalin, C. Brochier, G. Vistoli, B. L. Alan and P. Kozikowski, J. Am. Chem. Soc., 2010, 132, 10842; (b) J. A. Bergman, K. Woan, P. Perez-Villarroel, A. Villagra, E. M. Sotomayor and A. P. Kozikowski, J. Med. Chem., 2012, 55, 9891.

9 P. Kahnberg, A. J. Lucke, M. P. Glenn, G. M. Boyle, J. D. A. Tyndall, P. G. Parsons and D. P. Fairlie, J. Med. Chem., 2006, 49, 7611.

10 P. K. Gupta, R. C. Reid, L. Liu, A. J. Lucke, S. A. Broomfield, M. R. Andrews, M. J. Sweet and D. P. Fairlie, Bioorg. Med. Chem. Lett., 2010, 20, 7067.

11 B. Yoo and K. Kirshenbaum, Curr. Opin. Chem. Biol., 2008, 12, 714. 12 A. A. Grolla, V. Podestá, M. G. Chini, S. Di Micco, A. Vallario, A. A. Genazzani, P. L. Canonico, G. Bifulco, G. C. Tron, G. Sorba and T. Pirali, J. Med. Chem., 2009, 52, 2776.

13 D. Marcovici-Mizrahi, H. E. Gottlieb, V. Marks and A. Nudelman, J. Org. Chem., 1996, 61, 8402.

14 C. A. Sotriffer, H. Gohlke and H. Klebe, J. Med. Chem., 2002, 45, 1967.

15 J. Melesina, D. Robaa, R. J. Pierce, C. Romier and W. Sippl, J. Mol. Graphics Modell., 2015, 62, 342.

16 M. Guha, Nat. Rev. Drug Discovery, 2015, 14, 225. 